\title{
A Power Efficient Pilot Design for Multi-cell Massive MIMO Systems
}

\author{
Tuan Anh Le, Trinh Van Chien and Mohammad Reza Nakhai \\ Conference Paper
}

Cite this paper as:

Anh, T., Van, T., Reza, M. A Power Efficient Pilot Design for Multi-cell Massive MIMO Systems, In: 2018 IEEE GLOBAL CONFERENCE ON SIGNAL AND

INFORMATION PROCESSING (GLOBALSIP 2018). California: Institute of Electrical and Electronics Engineers (IEEE); 2018, pp. 823-827. ISBN: 978-1-7281-1295-4.

DOI: https://doi.org/10.1109/GlobalSIP.2018.8646568

The self-archived postprint version of this conference paper is available at Linköping University Institutional Repository (DiVA):

http://urn.kb.se/resolve?urn=urn:nbn:se:liu:diva-154875

Original publication available at:

DOI: https://doi.org/10.1109/GlobalSIP.2018.8646568

Copyright: Institute of Electrical and Electronics Engineers http://www.ieee.org/index.html (C)2018 IEEE. Personal use of this material is permitted. However, permission to reprint/republish this material for advertising or promotional purposes or for creating new collective works for resale or redistribution to servers or lists, or to reuse any copyrighted component of this work in other works must be obtained from the IEEE. 


\title{
A POWER EFFICIENT PILOT DESIGN FOR MULTI-CELL MASSIVE MIMO SYSTEMS
}

\author{
Tuan Anh Le $e^{\dagger}$, Trinh Van Chien ${ }^{\S}$, and Mohammad Reza Nakhai \\ ${ }^{\dagger}$ Department of Design Engineering \& Mathematics, Middlesex University, London, NW4 4BT, U. K. \\ ${ }^{\S}$ Department of Electrical Engineering (ISY), Linköping University, 58183 Linköping, Sweden. \\ ${ }^{\ddagger}$ Department of Informatics, King’s College London, London, WC2B 4BG, U. K. \\ Email:t.le@mdx.ac.uk; trinh.van.chien@liu.se; reza.nakhai@kcl.ac.uk
}

\begin{abstract}
In this paper, we address the pilot contamination problem in multi-cell massive MIMO systems. Particularly, we propose a pilot design scheme that simultaneously minimizes the channel estimation errors of all base stations (BSs) and the total pilot power consumption of all users subject to the transmit power constraint for every user in the network. We decompose the proposed non-convex problem into distributed optimization problems to be solved at each BS, assuming the knowledge of pilot signals of the other BSs. Then, we introduce a successive optimization approach to cast each distributed optimization problem into a convex linear matrix inequality form. Simulation results confirm that the proposed approach significantly reduces pilot power while maintain the same level of channel estimation error as a recent work in [1].
\end{abstract}

\section{INTRODUCTION}

Massive MIMO networks offer significant improvements in both energy and spectrum efficiency in comparison with conventional cellular networks [2]. The performance of a Massive MIMO system heavily depends on the accuracy of the channel sate information between its antennas and served users which is obtained via the pilot training phase. Unfortunately, due to the limit in the number of pilot signals available in a massive MIMO network, its performance severely suffers from pilot contamination [3]. To that end, several attempts have been made for pilot design which can be roughly classified into two main works: pilot assignment and pilot power control, see e.g., [4] and [5], respectively, and references therein. Recently, we studied a multi-cell massive MIMO system, where minimum mean square error (MMSE) estimators are employed at base stations (BSs) [1]. Based on the derived mean square error (MSE) formula of the employed MMSE estimator, we proposed a novel pilot design method to minimize the total MSE of all adopted-MMSE estimators subject to a power constraint at each user in the network.

The work of T. V. Chien was supported by the European Union's Horizon 2020 research and innovation programme under grant agreement No 641985 (5Gwireless), and also by ELLIIT and CENIIT.
Although the design can improve the accuracy of the channel estimator and the uplink spectral efficiency, power efficiency was not the focus of the method.

In this paper, we focus on two objectives: i) the total channel estimation errors at BSs; ii) the total pilot power consumed by all mobile users. While our previous work in [1] only considers the first objective, here, we simultaneously minimizes both objectives subject to a transmit power constraint for every user. The proposed new objective function is non-convex with respect to the optimization variables, i.e., matrices collecting all pilot signals. To tackle the problem, we introduce two auxiliary optimization variables for each cell and adopt the Schur complement [6] to transform the objective function into a linear form while moving the non-linear parts into two new constraints. Since one of the newly introduced constraints is non-convex, we continue by two following steps. First, we decompose the transformed optimization problem into distributed problems where each one will be solved at each cell based on the pilot signal knowledge of the other BSs. Second, we propose a successive optimization method to recast each distributed problem into a convex linear matrix inequality (LMI) problem. Finally, we provide a complexity analysis for the proposed LMI optimization problem.

Notation: Bold lower/upper case letters: vectors/matrices; $\|\cdot\|_{F}$ : the Frobenius norm; $\|\cdot\|$ : the Euclidean norm; $(\cdot)^{H}$ : complex conjugate transpose operator; $\operatorname{Tr}(\cdot)$ : the trace of a matrix; $\mathbf{W} \succeq \mathbf{0}$ : the positive semidefinite condition; $\mathbf{I}_{a}$ : an $a \times a$ identity matrix; $\operatorname{diag}\{\mathbf{w}\}:$ a diagonal matrix with the diagonal entries being elements of the vector $\mathbf{w} ;(\mathbf{A})^{-1}$ : the inverse of a square matrix; $(\mathbf{A})^{\frac{1}{2}}$ : the square root of a matrix; $\mathcal{C N}(\cdot, \cdot)$ : a circularly symmetric complex Gaussian distribution; $\mathbb{E}[\cdot]$ : the expectation of a random variable; $\mathcal{O}(\cdot)$ : the big-O notation.

\section{SYSTEM MODEL}

This paper investigates a time-division duplexing multi-cell massive MIMO system with $U$ cells where each comprising of an $M$-antenna BS and $N$ single-antenna users. The propagation coefficient between the $j$-th antenna of the BS in cell $q$, 
denoted as BS $q$, and user $m$ in cell $p$ is $\sqrt{\psi_{p m q}} h_{p m q j}$, where $\psi_{p m q}$ captures the large scale fading, i.e., path-loss and shadowing, while $h_{p m q j} \sim \mathcal{C N}(0,1)$ represents small-scale fading. Let $\mathbf{w}_{p m} \in \mathbb{C}^{\tau \times 1}$ denote the pilot signal of $\tau$ symbols transmitted by user $m$ in cell $p$ and $\left\|\mathbf{w}_{p m}\right\|^{2} \leq P_{\max , p}, \forall p$, where $P_{\max , p}$ is the maximum transmit power level of each user in cell $p$ allocated to its pilot signal. During the pilot training period, all users synchronously transmit their pilot signals. The received training signal $\mathbf{y}_{q j} \in \mathbb{C}^{\tau \times 1}$ at the $j$-th antenna element of $\mathrm{BS} q$ can be written as:

$$
\mathbf{y}_{q j}=\sum_{p=1}^{U} \sum_{m=1}^{N} \sqrt{\psi_{p m q}} h_{p m q j} \mathbf{w}_{p m}+\mathbf{n}_{q j},
$$

where $\mathbf{n}_{q j}$ denotes Gaussian noise with $\mathbf{n}_{q j} \sim \mathcal{C N}\left(\mathbf{0}, \sigma^{2} \mathbf{I}_{\tau}\right)$. In the following, we represent the received signals, Gaussian noises, pilot signals by all antenna elements of BS $q$ and the corresponding large scale channel coefficients, respectively, in matrix forms as

$$
\begin{aligned}
& \mathbf{Y}_{q}=\left[\mathbf{y}_{q 1}, \mathbf{y}_{q 2}, \ldots, \mathbf{y}_{q M}\right] \in \mathbb{C}^{\tau \times M}, \\
& \mathbf{N}_{q}=\left[\mathbf{n}_{q 1}, \mathbf{n}_{q 2}, \ldots, \mathbf{n}_{q M}\right] \in \mathbb{C}^{\tau \times M}, \\
& \mathbf{W}_{q}=\left[\mathbf{w}_{q 1}, \mathbf{w}_{q 2}, \ldots, \mathbf{w}_{q N}\right] \in \mathbb{C}^{\tau \times N}, \\
& \mathbf{\Psi}_{p q}=\operatorname{diag}\left\{\left[\psi_{p 1 q}, \psi_{p 2 q}, \ldots, \psi_{p N q}\right]^{T}\right\} \in \mathbb{C}^{N \times N} .
\end{aligned}
$$

We also stack all small-scale fading channel coefficients of $N$ users in cell $p$ as seen by BS $q$ in the following compact form

$$
\mathbf{H}_{p q}=\left[\begin{array}{ccc}
h_{p 1 q 1}, & \ldots, & h_{p 1 q M} \\
\vdots & \ddots & \vdots \\
h_{p N q 1}, & \cdots & , h_{p N q M}
\end{array}\right] \in \mathbb{C}^{N \times M} .
$$

From (1) - (6), we express the received training signals by all $M$ antenna elements of $\operatorname{BS} q$ as

$$
\mathbf{Y}_{q}=\mathbf{W}_{q} \mathbf{\Psi}_{q q}^{\frac{1}{2}} \mathbf{H}_{q q}+\sum_{p=1, p \neq q}^{U} \mathbf{W}_{p} \mathbf{\Psi}_{p q}^{\frac{1}{2}} \mathbf{H}_{p q}+\mathbf{N}_{q}
$$

The channel estimate $\widehat{\mathbf{H}}_{q q}$ of the original channel $\mathbf{H}_{q q}$ can be attained by adopting MMSE estimation [7]:

$$
\widehat{\mathbf{H}}_{q q}=\mathbb{E}\left[\mathbf{H}_{q q} \mathbf{Y}_{q}^{H}\right]\left(\mathbb{E}\left[\mathbf{Y}_{q} \mathbf{Y}_{q}^{H}\right]\right)^{-1} \mathbf{Y}_{q} .
$$

Let the channel estimation errors at BS $q$ be represented as $\mathbf{E}_{q}=\mathbf{H}_{q q}-\widehat{\mathbf{H}}_{q q}$. Then the MSE can be expressed as

$$
\operatorname{MSE}_{q}=\mathbb{E}\left[\left\|\mathbf{E}_{q}\right\|_{F}^{2}\right]=\mathbb{E}\left[\operatorname{Tr}\left(\mathbf{E}_{q} \mathbf{E}_{q}^{H}\right)\right] .
$$

Following the similar steps as in [1], one can reformulate (9) as

$$
f_{q}\left(\mathbf{W}_{q}\right)=M \operatorname{Tr}\left(\left(\mathbf{I}_{N}+\boldsymbol{\Psi}_{q q}^{\frac{1}{2}} \mathbf{W}_{q}^{H} \mathbf{L}_{q}^{-1} \mathbf{W}_{q} \mathbf{\Psi}_{q q}^{\frac{1}{2}}\right)^{-1}\right)
$$

where $\mathbf{L}_{q}=\sum_{p=1, p \neq q}^{U} \mathbf{W}_{p} \boldsymbol{\Psi}_{p q} \mathbf{W}_{p}^{H}+\sigma^{2} \mathbf{I}_{\tau}$.

\section{A POWER EFFICIENT PILOT DESIGN}

We introduce a pilot design to minimize the sum of the total channel estimation errors of all BSs and the total pilot power of all users in the network subject to the transmit power constraints at individual users as follows:

$$
\begin{array}{ll}
\underset{\left\{\mathbf{W}_{q}\right\}}{\operatorname{minimize}} & \sum_{q=1}^{U} f_{q}\left(\mathbf{W}_{q}\right)+\sum_{q=1}^{U} \tilde{\alpha}_{q} \sum_{m=1}^{N}\left\|\mathbf{w}_{q m}\right\|^{2} \\
\text { subject to } & \mathbf{W}_{q}^{H} \mathbf{W}_{q} \preceq P_{\max , q} \mathbf{I}_{N}, \forall q,
\end{array}
$$

where $\left\{\mathbf{W}_{q}\right\}=\left\{\mathbf{W}_{1}, \mathbf{W}_{2}, \cdots, \mathbf{W}_{U}\right\}$ and $\tilde{\alpha}_{q} \geq 0$ is the weighting factor for cell $q$ to balance between the channel estimation error and the total pilot power consumption from the $N$ users in this cell. ${ }^{1}$

The objective function of problem (11) is non-convex with respect to its optimization variables. To tackle the problem, we first introduce two auxiliary variables $\mathbf{Q}_{q}$ and $\mathbf{P}_{q}$ to shift the nonlinear part into constraints and hence transforming the objective function into a linear form. Denoting $\left\{\mathbf{Q}_{q}\right\}=\left\{\mathbf{Q}_{1}, \cdots, \mathbf{Q}_{U}\right\},\left\{\mathbf{P}_{q}\right\}=\left\{\mathbf{P}_{1}, \cdots, \mathbf{P}_{U}\right\}$, we can rewrite problem (11) in the epigraph form [6, pp.134] as

$$
\begin{array}{ll}
\underset{\left\{\mathbf{W}_{q}\right\},\left\{\mathbf{Q}_{q}\right\},\left\{\mathbf{P}_{q}\right\}}{\operatorname{minimize}} & \sum_{q=1}^{U} M \operatorname{Tr}\left(\mathbf{Q}_{q}+\frac{\tilde{\alpha}_{q}}{M} \mathbf{P}_{q}\right) \\
\text { subject to } & \mathbf{W}_{q}^{H} \mathbf{I}_{\tau}^{-1} \mathbf{W}_{q} \preceq P_{\max , q} \mathbf{I}_{N}, \forall q, \\
& \left(\mathbf{I}_{N}+\mathbf{\Psi}_{q q}^{\frac{1}{2}} \mathbf{W}_{q}^{H} \mathbf{L}_{q}^{-1} \mathbf{W}_{q} \mathbf{\Psi}_{q q}^{\frac{1}{2}}\right)^{-1} \preceq \mathbf{Q}_{q}, \forall q, \\
& \mathbf{W}_{q}^{H} \mathbf{I}_{\tau}^{-1} \mathbf{W}_{q} \preceq \mathbf{P}_{q}, \forall q .
\end{array}
$$

Denoting $\alpha_{q}=\tilde{\alpha}_{q} / M$, removing the constant $M$ and applying the Schur complement [6], we can equivalently recast problem (12) as

$$
\begin{array}{ll}
\underset{\left\{\mathbf{W}_{q}\right\},\left\{\mathbf{Q}_{q}\right\},\left\{\mathbf{P}_{q}\right\}}{\operatorname{minimize}} & \sum_{q=1}^{U} \operatorname{Tr}\left(\mathbf{Q}_{q}+\alpha_{q} \mathbf{P}_{q}\right) \\
\text { subject to } & {\left[\begin{array}{cc}
P_{\max , q} \mathbf{I}_{N} & \mathbf{W}_{q}^{H} \\
\mathbf{W}_{q} & \mathbf{I}_{\tau}
\end{array}\right] \succeq \mathbf{0}, \forall q,} \\
& {\left[\begin{array}{cc}
\mathbf{Q}_{q} & \mathbf{I}_{N} \\
\mathbf{I}_{N} & \mathbf{I}_{N}+\mathbf{\Psi}_{q q}^{\frac{1}{2}} \mathbf{W}_{q}^{H} \mathbf{L}_{q}^{-1} \mathbf{W}_{q} \boldsymbol{\Psi}_{q q}^{\frac{1}{2}}
\end{array}\right] \succeq \mathbf{0}, \forall q,} \\
& {\left[\begin{array}{cc}
\mathbf{P}_{q} & \mathbf{W}_{q}^{H} \\
\mathbf{W}_{q} & \mathbf{I}_{\tau}
\end{array}\right] \succeq \mathbf{0}, \forall q .}
\end{array}
$$

Since term $\boldsymbol{\Psi}_{q q}^{\frac{1}{2}} \mathbf{W}_{q}^{H} \mathbf{L}_{q}^{-1} \mathbf{W}_{q} \boldsymbol{\Psi}_{q q}^{\frac{1}{2}}$ is nonlinear with respect to the set of optimization variables $\left\{\mathbf{W}_{q}\right\}$, the second set of constraints in (13) is not convex. We take two steps to tackle the problem. Firstly, we introduce a distributed algorithm where each BS $q$ optimizes its own pilot signals given the knowledge

\footnotetext{
${ }^{1}$ Due to the space constraint, the treatment for $\tilde{\alpha}_{q}$ will be reported in our full report of this work.
} 
of the pilot signals of the other BSs in $\mathbf{L}_{q}^{-1}$ as follows:

$$
\begin{array}{cl}
\underset{\mathbf{W}_{q}, \mathbf{Q}_{q}, \mathbf{P}_{q}}{\operatorname{minimize}} & \operatorname{Tr}\left(\mathbf{Q}_{q}+\alpha_{q} \mathbf{P}_{q}\right) \\
\text { subject to } & {\left[\begin{array}{cc}
P_{\max , q} \mathbf{I}_{N} & \mathbf{W}_{q}^{H} \\
\mathbf{W}_{q} & \mathbf{I}_{\tau}
\end{array}\right] \succeq \mathbf{0},} \\
& {\left[\begin{array}{cc}
\mathbf{Q}_{q} & \mathbf{I}_{N} \\
\mathbf{I}_{N} & \mathbf{I}_{N}+\mathbf{\Psi}_{q q}^{\frac{1}{2}} \mathbf{W}_{q}^{H} \mathbf{L}_{q}^{-1} \mathbf{W}_{q} \mathbf{\Psi}_{q q}^{\frac{1}{2}}
\end{array}\right] \succeq \mathbf{0},} \\
& {\left[\begin{array}{cc}
\mathbf{P}_{q} & \mathbf{W}_{q}^{H} \\
\mathbf{W}_{q} & \mathbf{I}_{\tau}
\end{array}\right] \succeq \mathbf{0} .}
\end{array}
$$

Since $\mathbf{L}_{q}^{-1}$ is assumed to be known to $\mathrm{BS} q$, the distributed optimization problem (14) now only has $\mathbf{W}_{q}, \mathbf{Q}_{q}$, and $\mathbf{P}_{q}$ as optimization variables. However, its second constraint is still not in an LMI form with respect to $\mathbf{W}_{q}$. We take a second step by introducing a successive optimization approach where, at the $t$-th iteration, BS $q$ updates its pilot signals by solving the following distributed optimization problem:

$$
\begin{array}{ll}
\underset{\mathbf{W}_{q}^{(t)}, \mathbf{Q}_{q}^{(t)}, \mathbf{P}_{q}^{(t)}}{\operatorname{minimize}} & \operatorname{Tr}\left(\mathbf{Q}_{q}^{(t)}+\alpha_{q} \mathbf{P}_{q}^{(t)}\right) \\
\text { subject to } & {\left[\begin{array}{cc}
P_{\max , q} \mathbf{I}_{N} & \mathbf{W}_{q}^{(t), H} \\
\mathbf{W}_{q}^{(t)} & \mathbf{I}_{\tau}
\end{array}\right] \succeq \mathbf{0},} \\
& {\left[\begin{array}{cc}
\mathbf{Q}_{q}^{(t)} & \mathbf{I}_{N} \\
\mathbf{I}_{N} & \mathbf{I}_{N}+\mathbf{K}_{q}^{(t)}
\end{array}\right] \succeq \mathbf{0},} \\
& {\left[\begin{array}{cc}
\mathbf{P}_{q}^{(t)} & \mathbf{W}_{q}^{(t), H} \\
\mathbf{W}_{q}^{(t)} & \mathbf{I}_{\tau}
\end{array}\right] \succeq \mathbf{0},}
\end{array}
$$

where $\mathbf{K}_{q}^{(t)}=\boldsymbol{\Psi}_{q q}^{\frac{1}{2}} \mathbf{W}_{q}^{(t), H}\left(\mathbf{L}_{q}^{-1}\right)^{(t-1)} \mathbf{W}_{q}^{(t-1)} \mathbf{\Psi}_{q q}^{\frac{1}{2}}$, and $\mathbf{L}_{q}^{-1}$ obtained from the previous iteration is

$$
\left(\mathbf{L}_{q}^{-1}\right)^{(t-1)}=\sum_{p=1, p \neq q}^{U} \mathbf{W}_{p}^{(t-1)} \mathbf{\Psi}_{p q} \mathbf{W}_{p}^{(t-1), H}+\sigma^{2} \mathbf{I}_{\tau}
$$

$\mathbf{W}_{q}^{(t-1)}$ and $\mathbf{W}_{p}^{(t-1)}$ are the pilots of cells $q$ and $p$, respectively, attained from the $(t-1)$-th iteration. We have exploited the value of $\mathbf{W}_{q}^{(t-1)}$ to cast the second constraint of (15) in an LMI form with respect to both $\mathbf{W}_{q}^{(t)}$ and $\mathbf{Q}_{q}^{(t)}$. At the stationary point obtained after a sufficient number of iterations, the approximation $\mathbf{W}_{q}^{(t)} \approx \mathbf{W}_{q}^{(t-1)}, \forall q$, can be guaranteed with any desired accuracy. During initial iterations, the matrix on the left hand side of the second constraint of (15) is not Hermitian. This is due to the mismatch between $\mathbf{W}_{q}^{(t)}$ and $\mathbf{W}_{q}^{(t-1)}, \forall q$. To tackle the problem, we introduce a new optimization variable $\mathbf{V}_{q}^{(t)}$, i.e.,

$$
\begin{aligned}
2 \mathbf{V}_{q}^{(t)}= & \boldsymbol{\Psi}_{q q}^{\frac{1}{2}} \mathbf{W}_{q}^{(t), H}\left(\mathbf{L}_{q}^{-1}\right)^{(t-1)} \mathbf{W}_{q}^{(t-1)} \boldsymbol{\Psi}_{q q}^{\frac{1}{2}} \\
& +\boldsymbol{\Psi}_{q q}^{\frac{1}{2}} \mathbf{W}_{q}^{(t-1), H}\left(\mathbf{L}_{q}^{-1}\right)^{(t-1)} \mathbf{W}_{q}^{(t)} \boldsymbol{\Psi}_{q q}^{\frac{1}{2}}
\end{aligned}
$$

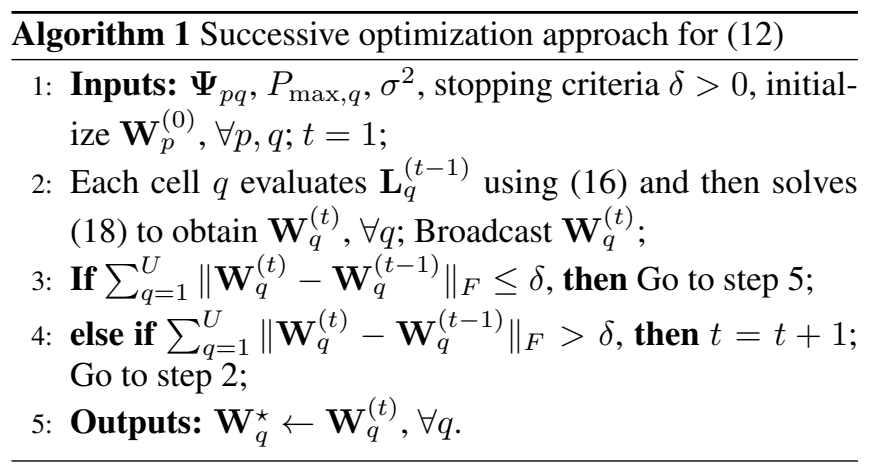

Finally, we equivalently cast (15) as the following LMI

$$
\begin{array}{ll}
\underset{\mathbf{W}_{q}^{(t)}, \mathbf{Q}_{q}^{(t)}, \mathbf{P}_{q}^{(t)}, \mathbf{V}_{q}^{(t)}}{\operatorname{minimize}} & \operatorname{Tr}\left(\mathbf{Q}_{q}^{(t)}+\alpha_{q} \mathbf{P}_{q}^{(t)}\right) \\
\text { subject to } & {\left[\begin{array}{cc}
P_{\max , q} \mathbf{I}_{N} & \mathbf{W}_{q}^{(t), H} \\
\mathbf{W}_{q}^{(t)} & \mathbf{I}_{\tau}
\end{array}\right] \succeq \mathbf{0},} \\
& {\left[\begin{array}{cc}
\mathbf{Q}_{q}^{(t)} & \mathbf{I}_{N} \\
\mathbf{I}_{N} & \mathbf{I}_{N}+\mathbf{V}_{q}^{(t)}
\end{array}\right] \succeq \mathbf{0},} \\
& {\left[\begin{array}{cc}
\mathbf{P}_{q}^{(t)} & \mathbf{W}_{q}^{(t), H} \\
\mathbf{W}_{q}^{(t)} & \mathbf{I}_{\tau}
\end{array}\right] \succeq \mathbf{0},} \\
& \text { constraint (17). }
\end{array}
$$

Problem (18) can be effectively solved by a standard interiorpoint method (IPM) $[6,8]$ provided by optimization packages such as CVX [9]. It can be observed that the number of decision variables of problem (18) is on the order of $(3 N+\tau) N$. Let $m=\mathcal{O}((3 N+\tau) N)$. Algorithm 1 summarizes the procedure to attain the optimal pilot signals for all $U$ cells in the network. The computational complexity of the major step of Algorithm 1 for any given $\epsilon>0$ is given by Lemma (1).

Lemma 1 The computational complexities to obtain $\epsilon$-solution to problem (18) is $\ln \left(\epsilon^{-1}\right) \sqrt{5 N+2 \tau} \beta m$, where $\beta=11 N^{3}+$ $(6 \tau+7 m) N^{2}+N \tau(6 \tau+4 m)+2 \tau^{2}(m+\tau)+m^{2}$.

Proof: Proof follows the similar steps as in $[1,10]$.

Since problem (18) is convex, steps 3 and 4 in Algorithm 1 ensure the $\delta$-convergence of $\mathbf{W}_{q}^{(t)}$ to its optimal value and a minimal objective function value in problem (18) per cell. Although solving (18) can yield the global optimality per iteration and per cell, it may not be the global optimal solution to the original multicell problem (11) due to its inherent nonconvexity. In fact, Algorithm 1 is a suboptimal algorithm with an affordable complexity to the NP-hard problem (11). Comparing the complexity of the proposed problem (18) and that of the problem in our previous work [1], one can conclude that the proposed problem has higher complexity than its counterpart does. However, the extra computation, which is affordable at BSs, results in reductions in pilot power, which is important for power-constrained mobile devices. 

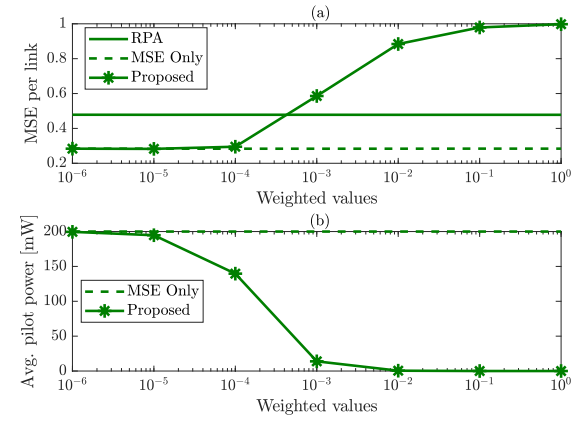

Fig. 1. Effects of the weight value.

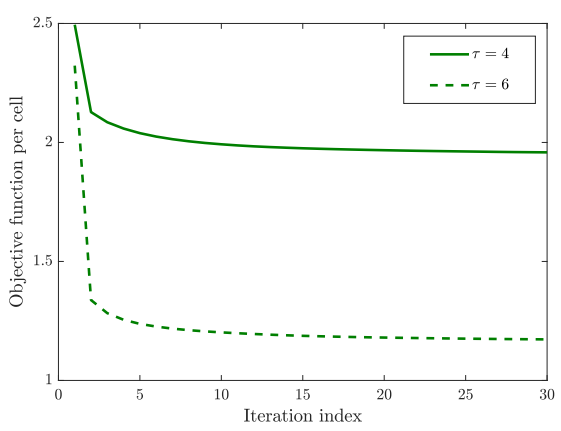

Fig. 2. Convergence of the proposed successive optimization.

\section{SIMULATION RESULTS}

A multi-cell Massive MIMO system with wrapped-around is considered for simulations with 4 cells where one BS equipped with 100 antennas is located at the center of each cell to serve 5 users. All users are randomly distributed over the coverage area under a condition that the distance between any user $m$ of cell $p$ and BS $q$, denoted as $d_{p m q}$ with $d_{p m q} \geq 0.035 \mathrm{~km}$. The network bandwidth is 20 $\mathrm{MHz}$. The noise variance is $-96 \mathrm{dBm}$ regarding to noise figure of $5 \mathrm{~dB}$. We model the large-scale fading coefficient as $\psi_{p m q}[\mathrm{~dB}]=-148.1-37.6 \log _{10}\left(d_{p m q}\right)+z_{p m q}$, where $z_{p m q}$ denotes the shadow fading which follows a log-normal Gaussian distribution with the standard variation of $7 \mathrm{~dB}$. Monte-Carlo simulations are carried over 200 independent realizations of user locations. Here, we compare the performance of our proposed pilot design with: $(i)$ The widely adopted orthogonal pilot design, e.g., $[3,5,11]$, where those orthogonal pilots are shared/reused amongst users in the network and a power of $200 \mathrm{~mW}$ is assigned for each pilot symbol. For each user location realization, such pilot signals are obtained by the eigenvectors of a uniformly generated random matrix. The power constraint for pilot signal is set to be $P_{\max , q}=200 \tau \mathrm{mW}, \forall q$. This random pilot assignment method is denoted as RPA in our figures. (ii) The mean-square-error only (MSE-Only) proposed in [1].

Figs. 1 (a) and (b), respectively, show the MSE per each BS-user link and the average pilot power, required to achieve that MSE level, versus different weighted values $\alpha_{q}$. Since
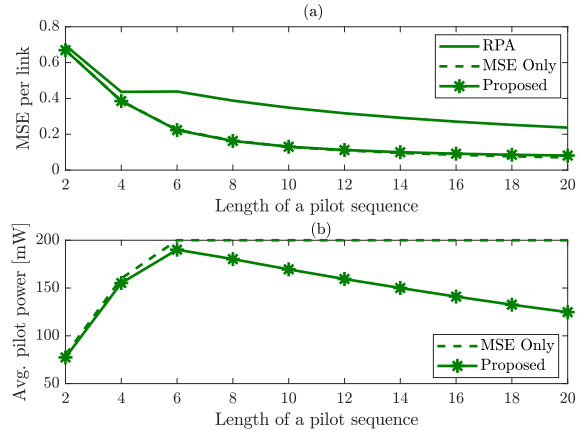

Fig. 3. Effects of the pilot length.

RPA always consume power $200 \mathrm{~mW}$ per pilot symbol, we do not include it in Fig. 1 (b). The figure reveals the tradeoff between the channel estimation accuracy and the average pilot power via the selection of $\alpha_{q}{ }^{2}$ For the $\alpha_{q}$ range from $10^{-6}$ to $10^{-4}$, the proposed approach attains the same MSE as our previous work in [1], however, at $\alpha_{q}=10^{-4}$, the proposed approach can save $30.2 \%$ on the pilot power consumption compared with its counterpart. Hereafter, unless otherwise stated, we set $\alpha_{q}=10^{-5}$ when qualifying problem (11) with different pilot lengths.

Fig. 2 displays the convergence of Algorithm 1 with two different pilot lengths. It is clear that the proposed solution method quickly converges with less than 20 iterations. Moreover, a large reduction in the objective function of problem (12) is observed when increasing the pilot length. For example, at the converged point, by adding two more symbols per pilot sequence, i.e., increasing $\tau$ from 4 to 6 , the the objective function of problem (12) can be further pushed down $23 \%$ thanks to more degrees of freedom are provided.

Figs. 3 (a) and (b), respectively, illustrate the channel estimation quality and the related pilot power consumption versus the length of a pilot sequence $\tau$. Fig. 3 (a) indicates that the proposed approach attains the same MSE level as the MSE-Only counterpart does while outperforming the RPA. Moreover, our proposed approach requires less pilot power than the MSE Only does, i.e., shown in Fig. 3 (b). The performance gap increases as the pilot length increases. For instance, at $\tau=2$, jointly optimizing the MSE and pilot power consumption only brings $3.39 \%$ power reduction while at $\tau=20$ doing so results in up to $37.62 \%$. Keeping in mind that 5 users are being served, it is interesting to observer from Fig. 3 (b) that the average pilot power significantly raises as the pilot length goes from 2 to 6 for both approaches. When the pilot length increases beyond 6, the MSE-Only approach requires all available power of $200 \mathrm{~mW}$ while the second objective in our proposed optimization problem can exploit higher degrees of freedom offered by more available orthogonal pilot sequences to further reduce pilot power.

\footnotetext{
${ }^{2}$ The range of $\alpha_{q}$ reflects the quantity differences between the two objectives and the fact that $M$ has been absorbed in it.
} 


\section{REFERENCES}

[1] H. Al-Salihi, T. V. Chien, T. A. Le, and M. R. Nakhai, "A successive optimization approach to pilot design for multi-cell Massive MIMO systems," IEEE Commun. Lett., vol. 22, no. 5, pp. 1086-1089, 2018.

[2] E. Björnson, E. G. Larsson, and T. L. Marzetta, "Massive MIMO: 10 myths and one critical question," IEEE Commun. Mag., vol. 54, no. 2, pp. 114 - 123, 2016.

[3] X. Zhu, Z. Wang, L. Dai, and C. Qian, "Smart pilot assignment for Massive MIMO," IEEE Commun. Lett., vol. 19, no. 9, pp. 1644 - 1647, 2015.

[4] S. Ma, E. L. Xu, A. Salimi, and S. Cui, "A novel pilot assignment scheme in Massive MIMO networks," IEEE Wireless Commun. Lett., vol. 7, no. 2, pp. 262$265,2018$.

[5] T. V. Chien, E. Björnson, and E. G. Larsson, "Joint pilot design and uplink power allocation in multi-cell Massive MIMO systems," IEEE Trans. Wireless Commun., vol. 17, no. 3, pp. $2000-2015$ s, 2018.

[6] S. Boyd and L. Vandenberghe, Convex Optimization. Cambridge University Press, 2004.

[7] S. Kay, Fundamentals of Statistical Signal Processing: Estimation Theory. Prentice Hall, 1993.

[8] K.-Y. Wang, A. M.-C. So, T.-H. Chang, W.-K. Ma, and C.-Y. Chi, "Outage constrained robust transmit optimization for multiuser miso downlinks: Tractable approximations by conic optimization," IEEE Trans. Signal Process., vol. 62, no. 21, pp. 5690-5705, 2014.

[9] CVX Research Inc., "CVX: Matlab software for disciplined convex programming, academic users," http://cvxr.com/cvx, 2015.

[10] T. V. Chien, E. Björnson, E. G. Larsson, and T. A. Le, "Distributed power control in downlink cellular Massive MIMO systems," in Proc. ITG Workshop on Smart Antennas (WSA), 2018.

[11] L. You, X. Gao, X.-G. Xia, N. Ma, and Y. Peng, "Pilot reuse for Massive MIMO transmission over spatially correlated rayleigh fading channels," IEEE Transactions on Wireless Communications, vol. 14, no. 6, pp. 33523366, 2015. 